\title{
A QoS-based User Reputation Measurement Method for Web Services
}

\author{
Jianlong $\mathrm{Xu}^{1,2,3, *}$, Yindong $\mathrm{Chen}^{1}$ and Changsheng $\mathrm{Zhu}^{1}$ \\ ${ }^{1}$ Shantou University, Shantou 515063, P.R. China \\ ${ }^{2}$ Hanshan Normal University, Chaozhou 521041, P.R. China \\ ${ }^{3}$ Guangdong Key Laboratory of Big Data Analysis and Processing, Guangzhou 510006, P.R. China \\ ${ }^{*}$ Corresponding author
}

\begin{abstract}
In Web services environment, high-quality services discovery and selection depend on reliable users with high reputations. However, user reputation is hard to accurately obtain directly. To evaluate the reliability of users, we present user reputation measurement method, named URMM. Based on the historical Quality of Service (QoS) values of users, we calculate user reputation value by the median and iteration operation. Based on real world public datasets, we conduct extensive experiments and demonstrate our proposed approach is more simple and effective than traditional approach.
\end{abstract}

Keywords-Web services; quality of service; reputation

\section{INTRODUCTION}

As a kind of distributed applications, Web services have played an important role in industry and academia [1], [2]. In Web services environment, users can invoke large amounts of Web services with equivalent similar functionalities. As the number of Web services increases on the Internet, it is necessary to select the best Web service that provides the best performance. To address this issue, many researchers have already carried out a great amount of work by employing personalized QoS properties (e.g., response time, invocation failure rate) which are observed by users [3], [4]. However, the most research work assumes that the QoS properties provided by users are reliable when making service selections. In fact, in the complex network environment, users may provide unreliable QoS values because: 1) The uses are irresponsible and may provide arbitrary QoS values, they may submit random or maximal / minimal values as their service QoS evaluation data. 2) Some users may be the service providers simultaneously, so they may give good QoS values for their own services and bad QoS values for their competitors' services. Therefore, unreliable users have a strong impact on the services selection. If users select services according to the QoS data given by unreliable users, they may select the unsuitable or bad services. User reputation needs to be considered when making selection and accurate reputation measure of Web services is crucial.

To address this problem above, in this paper, we present user reputation calculation model, named URMM. In our approach, reputation measurement model is based on the historical QoS values. Our major contributions are summarized as follows: 1) We present URMM to calculate the reputation of each user. Based on the historical QoS values, our model can provide reasonable reputation value for each user. 2) Both theoretical analysis and extensive experiments are conducted to demonstrate that our model is more simple and effective in contrast to traditional approach.

The rest of our paper is organized as follows. We review the related work in Section 2 and propose our reputation model in Section 3. Section 4 presents experimental results and Section 5 concludes the paper.

\section{RELATED WORK}

User reputation indicates the reliability and creditability of a user. Since the variability and uncertainty of user behavior, user reputation is hard to accurately obtain directly. Therefore reputation calculation models have been attracted a great deal of attention by many researchers. Generally, reputation calculation methods can be divided into two categories: content-driven and user-driven. For content-driven, reputation value is obtained according to the quality and quantity of usergenerated contents and the survival time of these contents, such as Wikipedia entry [5] and the location edit of the Google map system [6]. For user-driven, the user reputation calculation and analysis are based on user feedback ratings. Obviously, the user reputation calculation of Web services belongs to user-driven. In the related researches, Mizzaro et al. [7] proposed Mizz model which was applied in assessment of scholarly papers. But this model cannot guarantee the convergence of reputation calculation. De et al. [8] proposed an iterative filtering method from an optimization perspective, but the convergence of the algorithm is affected by the parameter settings. To address the convergence issue, Rong-Hua $\mathrm{Li}$ et al. [9] put forward six convergence algorithms for reputation calculation, there are L1AVG, L2AVG, L1MAX, L2MAX, L1MIN and L2M1N, respectively. Based on the work of Rong-Hua Li, Baichuan Li et al. [10] propose a topic-biased model (TBM) to estimate user reputation which is applied in rating systems. However, these two models are also affected by the parameter settings, such as the damping factor.

Our work is inspired by [9] and [10], which focuses on the convergence of the algorithm. Different from the related work, our algorithm provides more simple and direct way to obtain the user reputation which does not adjust the parameter in the calculative process. In addition, experiment on real data shows that our model is more effective. 


\section{MODEL}

\section{A. Problem Description}

In Web services environment, service invocations can produce a user-service QoS matrix with respect to each QoS attribute. In this matrix, each row and column denotes a service user and a candidate service, respectively, and each entry in the matrix denotes the QoS value observed by a user when invoking a service. However, in contrary to the rating values in rating systems, the QoS values are not limited in a certain range. Therefore, traditional reputation calculation models may not be applicable.

We assume a service computing environment with $m$ users $U=\left\{u_{1}, u_{2}, \ldots, u_{\mathrm{m}}\right\}$ and $n$ services $S=\left\{s_{1}, s_{2}, \ldots, s_{\mathrm{n}}\right\}$. The userservice matrix is an $m \times n$ matrix $Q \in \mathrm{R}^{m \times n}$, each entry in this matrix $q_{i j}(i \leq m, j \leq n)$ denotes the value of QoS property (e.g., response time, throughput) of Web service $j$ observed by service user $i$. If user $i$ did not invoke Web service $j$ before, then $q_{i j}=$ null. Our goal of reputation calculation is to excavate the information from the QoS data of each user.

\section{B. User Reputation Calculation Model}

Literature [9] had proposed L2-AVG method to calculate reputation which can be express as:

$$
\left\{\begin{array}{l}
A_{j}^{k+1}=\frac{1}{\left|H_{j}\right| \sum_{u_{j} \in I_{j}} q_{i j} r_{i}^{k}} \\
r_{i}^{k+1}=1-\frac{d}{\left|O_{i}\right|} \sum_{s_{j} \in O_{i}}\left(q_{i j}-A_{j}^{k+1}\right)^{2}
\end{array}\right.
$$

where $A_{j}$ is the average QoS value for service $j, k$ indicates the $k^{\text {th }}$ iteration, $A_{j}^{k+1}$ is $A_{j}$ in the $k+1^{\text {th }}$ iteration, $r_{i}^{k}$ is the reputation $r_{i}$ in the $k^{\text {th }}$ iteration, $H_{j}$ is the set of users which invokes service $j,\left|H_{j}\right|$ denotes the quantity of users who have invoked service $j, O_{i}$ is the set of services invoked by user $i,\left|O_{i}\right|$ denotes the number of services which have been invoked by user $i, d$ is a decay constant in $(0,1)$.

The main idea of L2-AVG algorithm is to find out the degree of user QoS value deviates from the population mean. However, it is suitable for rating system that the rating values are limited in a certain range. In real-world, since the distributions of QoS data are highly skewed with large variances, this method may suffer when users give unreliable ratings. For example, an unreliable user gives a negative date or very big date may have a negative impact on the mean and the reputation calculation results. To address this problem, we propose our new user reputation evaluation approach based on medium value analysis, which named URMM.

We assume the reputation of users can be represented as $R=\left\{r_{1}, r_{2}, \cdots, r_{\mathrm{m}}\right\}\left(0 \leq r_{i} \leq 1\right)$. The most reliable user's reputation value is 1 while the least reliable user's reputation value is 0 . We have:

$$
\left\{\begin{array}{l}
M_{j}^{k+1}=\underset{u_{i} \in H_{j}}{\operatorname{med}}\left(q_{i j} r_{i}^{k}\right) \\
r_{i}^{k+1}=1-\frac{1}{\left|O_{i}\right|} \sum_{\mathrm{s}_{j} \in O_{i}}\left(\frac{\left(q_{i j}-M_{j}^{k+1}\right)^{2}}{\max _{\mathrm{s}_{j} \in O_{i}}\left(q_{i j}-M_{j}^{k+1}\right)^{2}}\right)
\end{array}\right.
$$

where $M_{j}$ is the medium QoS value for service $j, M_{j}^{k+1}$ is $M_{j}$ in the $k+1^{\text {th }}$ iteration, the meaning of other parameters $r_{i}^{k}, H_{j}, O_{i}$, $\left|O_{i}\right|$ are the same with Eq.(1).

The calculation method of Eq.(2) can be explained that a certain user reputation depends on the gap between QoS values observed by this user and the medium of QoS values observed by other users. If a user provides a Web service QoS values which are quite difference with medium of other users' QoS values, then the user is likely to be unreliable. In Eq.(2), $r_{i}$ is determined by $q_{i j}$ and $M_{j}^{k+1}$. In our model, in the initialization step, $k=0$ and $r_{i}^{o}=1$. Then the medium QoS value for service $j$ and the reputation of the user $i$ are calculated according to Eq. (2) by an iterative method. When $k$ is more than RMaxI (the maximum number of iterations) or the absolute of $r_{i}^{k+1}-r_{i}^{k}$ satisfies the required accuracy (less than threshold), the algorithm will be terminated and outputs the user reputation.

\section{EXPERIMENT}

In this section, we conduct experiments to validate our URMM approach. We use the real-world datasets released by Zheng et al. [11] in our experiments. This dataset includes a $339 \times 5825$ matrix containing 339 service users and 5825 real Web services. The entries in the matrix include response time property and throughput. In our experiments, we adopt a part of the response time dataset and add unreliable user to the dataset. The response time values of the unreliable users are randomly generated and these values are unreliable. We use a $5 \times 6$ userservice matrix where data of user 1 to 4 are reliable and user 5 is unreliable. The goal of our experiment is to calculate each user's reputation value and identify the unreliable user by our approach. In the following experiments, we set RMaxI as 10 and set threshold as 0.001 . Table 1 show the user-service matrix.

TABLE I. $5 \times 6$ USER-SERVICE RESPONSE TIME MATRIX

\begin{tabular}{lcccccc}
\hline & Service & Service & Service & Service & Service & Service \\
& 1 & 2 & 3 & 4 & 5 & 6 \\
\hline User 1 & 5.9820 & 0.2280 & 0.2370 & 0.2210 & 0.2220 & 0.5270 \\
User 2 & 2.1300 & 0.2620 & 0.2730 & 0.2510 & 0.2540 & 0.4270 \\
User 3 & 0.8540 & 0.3660 & 0.3760 & 0.3570 & 0.3580 & 0.1150 \\
User 4 & 0.6930 & 0.2260 & 0.2330 & 0.2200 & 0.2190 & 0.3440 \\
User 5 & 40.2633 & 43.6892 & 41.3455 & 42.1141 & 42.7393 & 44.7136 \\
\hline
\end{tabular}

To prove the effectiveness of our method, we ran extensive experiments and compare our method with L2-AVG [9]. We vary decay constant $d$ with different value for L2-AVG. The experimental results of users reputation calculation iteration process based on L2-AVG is illustrated in Figure 1.

From Figure 1, when $d=0.0001$, the reputations of user 1 to 5 are $0.9957,0.9953,0.9950,0.9950,0.8793$, respectively. 
When $d=0.001$, the reputations of user 1 to 5 are 0.9979 , $1.0000,0.9998,0.9998,-0.7853$, respectively. The reputation values are different with different decay constant. When $d=0.001$, the reputations value of user 5 is negative, which contradicts the range of reputation. The reason is that: in the process of iterative, if the decay constant $d$ is inappropriate, the value of multiplying $\left(q_{i j}-A_{j}^{k+1}\right)^{2}$ and $d$ will be increased by the QoS date of user 5 in Eq.(1), and the result of dividing $\left|\boldsymbol{O}_{i}\right|$ by this value is bigger than 1 . Therefore, it needs to adjust $d$ to obtain satisfactory results when L2-AVG algorithm is employed.

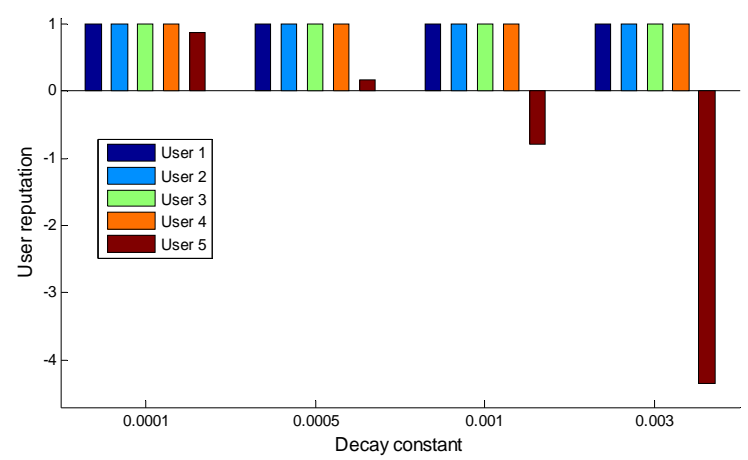

(a) Different decay constant for L2-AVG

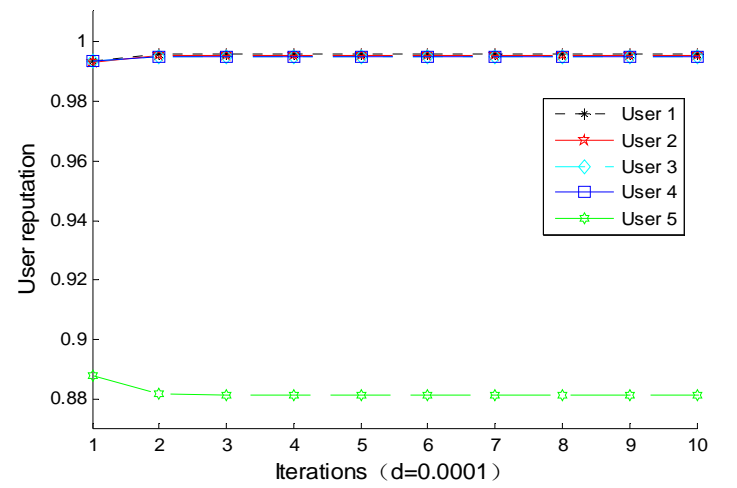

(b) $\mathrm{L} 2-\mathrm{AVG}(\mathrm{d}=0.0001)$

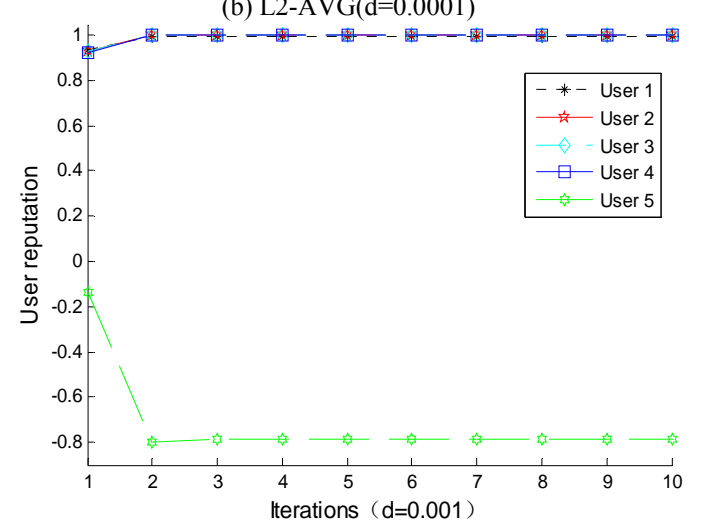

(c) $\mathrm{L} 2-\mathrm{AVG}(\mathrm{d}=0.001)$

FIGURE I. USER REPUTATION CALCULATION ITERATION PROCESS BASED ON L2-AVG

The experimental results of our approach are illustrated in Figure 2. Figure 2 (b) is the enlarged view of user 1 to user 4 in
Figure 2 (a). From Figure 2, the reputations of user 1 to 5 are $0.9999,1.0000,1.0000,0$, respectively. Comparing with the L2-AVG algorithm, our approach is more simple and efficient. Firstly, it needs no decay constant to adjust the calculation result. Second, for L2-AVG algorithm, the average value is tremendously impacted by the specific abnormal data (e.g. the data of user 5) while our approach can avoid being impacted by the specific abnormal data. When suffering from an unreliable user (the QoS data $q_{i j}$ is very big or very small relative to other data of reliable user), since $\left(q_{i j}-M_{j}^{k+1}\right)^{2}$ is close to or equal to $\max \left(q_{i j}-M_{j}^{k+1}\right)^{2}$, the reputation of unreliable user is very small in our approach. Third, our model is faster than L2-AVG $(\mathrm{d}=0.0001)$, the reputations can be obtained after two iterations in our algorithm while three iterations in L2-AVG when $\mathrm{d}=0.0001$.

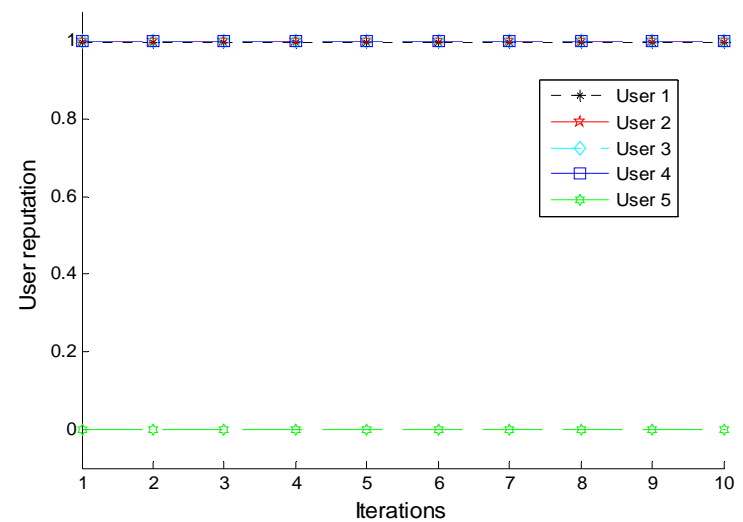

(a) The reputation values of user 1 to 5

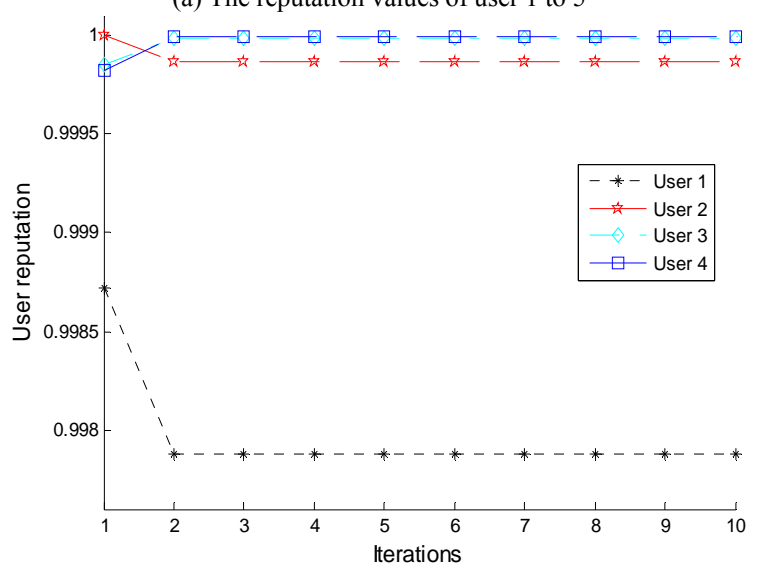

(b) The reputation values of user 1 to 4

FIGURE II. USER REPUTATION CALCULATION ITERATION PROCESS BASED ON URMM

\section{CONCLUSION AND FUTURE WORK}

It is essential to obtain user reputation value before Web services selection and recommendation. In this paper, we present a user reputation calculation approach, namely URMM, which is based on the median and iteration operation. Extensive experiments are conducted on a real-world dataset and the experimental results show that our proposed approach is more simple and effective in contrast to traditional approach. In the future work, we aim to investigate better user reputation 
calculation approaches, for example, we will try to optimize the reputation calculating method by taking the online environment into consideration, do more experiments to test the performance of our model and compare with more other algorithms.

\section{ACKNOWLEDGMENT}

This research was financially supported by the National Natural Science Foundation of China (No. 61702318), the Guangdong High-Level University Project "Green Technologies for Marine Industries", Guangdong Common Colleges Young Innovative Talents Project (No. 2016KQNCX056), and Shantou University National Fund breeding project (No.NFC16001), Guangdong Natural Science Foundation (2015A030313433), Characteristic Innovation project in Higher Education of Guangdong (2015KTSCX036).

\section{REFERENCES}

[1] Martin Garriga, Andres Flores, Alejandra Cechich, Alejandro Zunino, "Web Services Composition Mechanisms: A Review," IETE Technical Review, vol.32, 2015, pp. 376-383.

[2] Jianlong Xu, Zibin Zheng, and Michael R. Lyu. "Web Service Personalized QoS Prediction via Reputation-based Matrix Factorization". IEEE Transactions on Reliability. vol.65, 2016, pp.28-37.

[3] Bernard O, Paul S, Beat W, "Modeling Services and Web Services: Application of ModelBus," In Proceedings of the International Conference on Software Engineering Research and Practice, Las Vegas, 2017, pp. 557-563

[4] Jianxun Liu , Mingdong Tang , Zibin Zheng. "Location-Aware and Personalized Collaborative Filtering for Web Service Recommendation," IEEE Transactions on Services Computing, vol.9, 2016, pp. 686-699.

[5] B. Thomas Adler, Luca de Alfaro, "A content-driven reputation system for the Wikipediat," In Proceedings of the 16th ACM International Conference on World Wide Web, Banff, Canada, 2007, pp. 261-270.

[6] Adler, B. T., De, A. L., Kulshreshtha, A., and Pye, I, "Reputation systems for open collaboration," Communications of the ACM, vol. 54, 2011, pp. 81-87.

[7] Mizzaro S. "Quality control in scholarly publishing: A new proposal," Journal of the American Society for Information Science and Technology, vol.54, 2003, pp. 989-1005.

[8] De Kerchove C, Van Dooren P. "Iterative filtering in reputation systems," SIAM Journal on Matrix Analysis and Applications, vol.31, 2010, pp. 1812-1834.

[9] Rong-Hua Li, Jerry $\mathrm{Yu}$ Xu, Huang Xin, Hong Cheng. "Robust Reputation-Based Ranking on Bipartite Rating Networks," In Proceedings of the 2012 SIAM International Conference on Data Mining, 2012, pp.612-623.

[10] Baichuan Li, Rong-Hua Li, Irwin King, M.R. Lyu, and Jeffrey Xu Yu. "A Topic-Biased User Reputation Model in Rating Systems," Knowledge and Information Systems (KAIS), vol.44, 2015, pp. 581-607.

[11] Zibin. Zheng, Yilei. Zhang, and Michael. R. Lyu, "Distributed QoS evaluationfor real-world web services," in Proceedings of the 17th IEEE International Conference on Web Services (ICWS), Miami, Florida, 2010, pp. 83-90. 